\title{
Depth dependent properties of ITO thin films grown by pulsed DC sputtering
}

\author{
A. Sytchkova ${ }^{\mathrm{a}, *}$, D. Zola ${ }^{\mathrm{a}}$, L.R. Bailey $^{\mathrm{b}}$, B. Mackenzie ${ }^{\mathrm{b}}$, G. Proudfoot $^{\mathrm{b}}$, M. Tian $^{\mathrm{c}}$, A. Ulyashin $^{\mathrm{d}}$ \\ a ENEA Optical Coatings Laboratory, via Anguillarese 301, 00123 Rome, Italy \\ b Oxford Instruments Plasma Technology, Yatton, Bristol, BS49 4AP, UK \\ c NT-MDT Europe BV, High Tech Campus 83, 5656 AG Eindhoven, The Netherlands \\ d SINTEF Materials and Chemistry, Forskningsveien 1, P.O. 124 Blindern, NO-0314 Oslo, Norway
}

\section{A R T I C L E I N F O}

\section{Article history:}

Received 7 July 2012

Received in revised form 13 October 2012

Accepted 25 November 2012

Available online 20 December 2012

\section{Keywords:}

ITO

Ellipsometry

Solar cells

\begin{abstract}
A B S T R A C T
A systematically prepared set of ITO layers for solar cell applications has been analyzed by spectroscopic variable angle ellipsometry in order to trace the dependence of free carriers' distribution along the film depth as a function of film thickness as well as its change upon annealing. Samples were deposited on silicon substrates with various thicknesses in steps of approximately $10-20 \mathrm{~nm}$. This set was duplicated and these samples were annealed, so that for each thickness an as-deposited and an annealed sample is available. Conventionally measured electrical conductivity and morphological properties (AFM measurements) of the films have been compared with the optical constants' inhomogeneity, i.e. material properties along the film thickness modelled by variable-angle spectroscopic ellipsometry. The obtained results show that the optical as well as electrical properties of thin ITO films prepared by pulsed DC sputtering are depth dependent. For the deposition conditions used a well-determined reproducible non-uniform distribution of free carriers within the film thickness was determined. In particular it has been found that the majority of free carriers in as-deposited ultra-thin ITO films is concentrated at sample half-depth, while their distribution becomes asymmetric for the thicker films, with a maximum located at approximately $40 \mathrm{~nm}$ depth. The distribution of free carriers in annealed samples is qualitatively different from that of as-deposited layers.
\end{abstract}

(c) 2012 Elsevier B.V. All rights reserved.

\section{Introduction}

Indium tin oxide (ITO) continues to be the material of choice for many applications where highly conductive thin films of high transparency are required: flat panel displays, solar cells, defrosting windows, electromagnetic shielding etc. [1-3]. It is one of the most studied transparent conducting oxides (TCOs) in terms of dependencies of its optical, electrical, morphological and structural properties on deposition conditions by various methods (see for example [2] for the history of processes for making TCOs) as well as on post-deposition treatment like annealing, e.g. [4], or plasma hydrogenation [5]. Incomparable combination of good optical and electrical performance of this material may be properly tuned, according to an application demands, by variation of deposition conditions and by the subsequent treatment.

Carrier transport in TCOs have been investigated in a series of works modelling the conductivity mainly based on the Hall and Seebeck measurements, e.g. [6,7] that provide the values for carrier concentration and mobility in the film. Such modelling combines compensated electron conduction within the film crystallites and

\footnotetext{
* Corresponding author.

E-mail address: anna.sytchkova@enea.it (A. Sytchkova).
}

thermoionic emission of carriers across the grain boundaries [8]. While scattering from grain boundaries and ionized impurities is the dominant free carriers' mobility limitation in materials with relatively low lattice mobility, for ITO these two scattering processes should be accomplished by other scattering mechanisms, e.g. [9]. Furthermore, for heavily doped semiconductors like $10 \%$ doped $\mathrm{In}_{2} \mathrm{O}_{3}$, one deals with a mixture of phases with different conductivities, whereas in the case of a thin film a contribution from different structural material organizations, amorphous and crystalline, should be also considered [10]. All these factors determine the overall trap density that is the measure of TCO electrical performance. Recently, for polycrystalline magnetron sputtered ITO, a significant variation of the mobilities in the carrier concentration range $N>3 \times 10^{20} \mathrm{~cm}^{-3}$ was found and a phenomenological model was created for temperature-dependent mobility of carriers that combines a temperature-independent term with a metal-like contribution, i.e. a thermally activated part due to grain boundarylimited transport [9]. In the cited work an attempt was done moreover to find a correlation between the discharge voltages and the trap densities for different deposition configurations of sputtering, including DC or RF excitations and different RF frequencies. While such a correlation was established for the other of two studied materials, no correlation was found for ITO, despite the well-known significant influence of reactive oxygen on the TCO growth and properties, e.g. [11]. 
Table 1

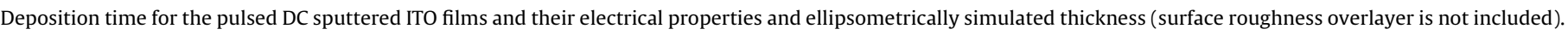

\begin{tabular}{|c|c|c|c|c|}
\hline Sample number & Deposition time & Resistivity $(\mu \Omega \mathrm{cm})$ & $\begin{array}{l}\text { Resistivity after anneal } \\
(\mu \Omega \mathrm{cm})\end{array}$ & $\begin{array}{l}\text { Film thickness, ellips } \\
\text { modelled (nm) }\end{array}$ \\
\hline 241 & $1 \mathrm{~min} 36 \mathrm{~s}$ & 413 & 297 & 78.4 \\
\hline 242 & $1 \mathrm{~min} 24 \mathrm{~s}$ & 415 & 273 & 67.8 \\
\hline 243 & $1 \min 12 \mathrm{~s}$ & 434 & 247 & 58.6 \\
\hline 244 & $1 \mathrm{~min} 00 \mathrm{~s}$ & 441 & 241 & 48.7 \\
\hline 245 & $0 \min 48 \mathrm{~s}$ & 465 & 235 & 40.9 \\
\hline 246 & $0 \min 36 s$ & 512 & 346 & 30.3 \\
\hline 247 & $0 \mathrm{~min} 24 \mathrm{~s}$ & 601 & 597 & 19.4 \\
\hline 248 & $0 \min 12 \mathrm{~s}$ & 1005 & 892 & 10.9 \\
\hline 249 & $0 \min 12 \mathrm{~s}$ & 1320 & & 9.5 \\
\hline 250 & $0 \min 24 \mathrm{~s}$ & 689 & & 18.2 \\
\hline 251 & $0 \min 36 s$ & 533 & & 28.4 \\
\hline 252 & $0 \min 48 \mathrm{~s}$ & 474 & & 38.3 \\
\hline 253 & $1 \mathrm{~min} 00 \mathrm{~s}$ & 447 & & 47.9 \\
\hline 254 & $1 \min 12 \mathrm{~s}$ & 428 & & 57.4 \\
\hline 255 & $1 \mathrm{~min} 24 \mathrm{~s}$ & 403 & & 65.9 \\
\hline 256 & $1 \mathrm{~min} 36 \mathrm{~s}$ & 420 & & 76.5 \\
\hline
\end{tabular}

The values of carrier concentration and mobility are characteristics of the film as a whole, i.e. in the mentioned models the film material is considered homogenous. Little is known about the internal organization of free carriers within the film thickness and in general about thickness dependence of ITO optical and electrical properties. Information on the carrier distribution within the film depth is useful for several reasons like, for example, adjustment of the deposition process parameters or those of post-deposition treatment, as well as minimization of the material use once an optimal film thickness with a sufficient number of charge carriers is found.

A study on the microstructure and electrical properties thickness dependence for ITO film deposited by RF magnetron sputtering on plastic substrate is reported in Ref. [12]. The study was performed on a set of samples with thicknesses varying in the range $40-280 \mathrm{~nm}$ (measured by a profilometer), and changes in microstructural and morphologic properties were observed using a scanning electron microscope. No optical characterization of the film properties was performed, or any study of carrier concentration versus film thickness variation.

Recently, an ellipsometric study of ITO inhomogeneity was performed for the RF sputtered films of approximately $1 \mu \mathrm{m}$ thickness [13]. The geometrical variation of the refractive index of the material, and in particular its imaginary part, i.e. the extinction coefficient, was noticed as an indicator of free carrier distribution along the film depth. The effects of deposition temperature and post-deposition annealing on re-distribution of the carriers were traced as well. Moreover, the cited study has underlined the importance of the film inhomogeneity modelling for a correct determination of the film thickness. In fact, inadequate characterization yielding imprecise film thickness values preclude correct determination of charge carrier density, mobility, the effective electron mass, the scattering mechanisms and relaxation time [3]. Such imprecision in film thickness value determination may be either due to a poor accuracy of the measurement technique, like that of mechanical profilometry, or due to deficient modelling of the indirect measurements like modelling of optical spectra without film inhomogeneity.

In this work we present a methodical study of optical, electrical and morphological properties of ITO performed on a set of layers with thickness varying from 10 to $80 \mathrm{~nm}$, the thickness mostly useful for solar cell applications. The insight into film properties change with its growth is achieved thanks to observation of a systematic law of the film refractive index inhomogeneity studied together with the thickness dependence of the layer morphology and resistivity. Moreover, film surface modelling by a porous overlayer for ellipsometric data is shown to be a reliable indicator of the film roughness obtained by the AFM technique.

\section{Experimental details}

The samples were deposited on one-side polished crystalline $\langle 100\rangle$ p-doped silicon substrates using a demonstration pulsed DC sputtering tool PlasmaPro System 400 (Oxford Instruments Co.). Depositions were carried out in a reactive gas chemistry and precision controlled $\mathrm{O}_{2}$ starting from a $200 \mathrm{~mm}$ diameter target of 99.99\%-pure ITO $\left(\mathrm{SnO}_{2}: \mathrm{In}_{2} \mathrm{O}_{3} 10: 90 \% \mathrm{wt}\right)$ equipped with a magnetron, $290 \mathrm{~W}$ DC power with a pulse frequency of $100 \mathrm{kHz}$. The processing pressure at the start and during deposition was $2 \mathrm{~m}$ Torr. The percentage of oxygen to argon was $2 \%$. From one deposition run to another only the film thickness, that is the run time, was varied with a step of $12 \mathrm{~s}$ which corresponds to about $10 \mathrm{~nm}$. Half of the samples were then annealed at $160^{\circ}$ for $2 \mathrm{~h}$ in air. Our research found that annealing ITO at $160^{\circ} \mathrm{C}$ for $2 \mathrm{~h}$ was equivalent to annealing at $300^{\circ} \mathrm{C}$ for $1 \mathrm{~h}$. The lower temperature anneal was utilized since there are applications where device damage could result in using higher temperatures. Table 1 summarizes the deposition times and reports the film resistance values obtained by van der Pauw four-point technique on twin samples deposited during the same runs on glass.

The samples were measured with a variable angle spectroscopic ellipsometer (WVASE, J.A. Woollam Co.) in the spectral range of $300-2500 \mathrm{~nm}$. At least three incidence angles were used for this analysis, $55^{\circ}, 65^{\circ}$ and $75^{\circ}$, while the thinnest layers were additionally checked at a grazing angle of $85^{\circ}$.

All the samples were investigated by atomic force microscopy (AFM) on an Ntegra integrated system (NT-MTD Co.). AFM measurements were carried out in semi-contact mode using a standard silicon probe. The probes were used with a spring constant of $\sim 2.5 \mathrm{~N} / \mathrm{m}$ and a free resonance of $\sim 150 \mathrm{kHz}$. The silicon tip at the end of these AFM probes had a curvature of $\sim 10 \mathrm{~nm}$. Both topography and phase images were recorded simultaneously in the scanning areas of $5 \mu \mathrm{m} \times 5 \mu \mathrm{m}$ and $1 \mu \mathrm{m} \times 1 \mu \mathrm{m}$. All the images were collected in air at scan rate of $1.0 \mathrm{~Hz}$ with $600 \times 600$ pixels resolution. AFM images were analyzed by using Nova Px software (NT-MDT Co.) yielding surface roughness parameters of 'Root Mean Square (RMS) roughness' $\left(R_{\mathrm{q}}\right)$ and average roughness $\left(R_{\mathrm{a}}\right)$.

\section{Ellipsometric analysis}

The ITO material optical dispersion was described by a general oscillator model containing three different oscillators: one 
Tauc-Cauchy that is representative for the UV-vis part of the acquired spectrum and two Lorentz ones which express the NIR optical behaviour of the material. The two Lorentz oscillators were necessary to accurately reproduce the shape of the curves of the ellipsometric angles, most probably due to a slight asymmetry of the absorption band in the NIR. In fact one of them is much less intensive and broader than the other. Notice that use of an extended Drude model (EDM) is another valid way for describing the ITO film transport processes [14-16], in particular because it accounts for ionized impurity scattering. Typically, EDM is used to correct Drude approximation for interband transitions, which in practice means additional Lorentz-type oscillator(s) into the original Drude model. The choice of the dispersion model should not however influence the conclusions on film inhomogeneity as each of the models leads to a reasonable fit of experimental data within reliable values of the optical constants.

The overall dispersion of the material was then considered smoothly variable along the layer depth using a three-pole grading. A higher polynomial order was inefficient as it would not

\begin{tabular}{|c|}
\hline Overlayer with $50 \%$ voids \\
\hline Graded ITO \\
\hline $\mathrm{SiO}_{2}$ native oxide $1.63 \mathrm{~nm}$ \\
\hline Si substrate $0.5 \mathrm{~mm}$ \\
\hline
\end{tabular}

Fig. 1. Schematic presentation of the layered model for ellipsometric characterization of the ITO/Si samples.

correspond to any physical process of film growth, while from a mathematical point of view this would add one more fit parameter and hence, increase instability of the inverse problem of film characterization. Both the refractive index $n$ and the extinction coefficient $k$ of the film are functions that may be considered a product of their dispersion and geometrical dependencies:

$$
n(\lambda, z)=n(\lambda) q(z) ; \quad k(\lambda, z)=k(\lambda) q(z)
$$

where $\lambda$ is the test wavelength and $0<q(z) \leq 1$ is the film profile function describing the optical functions' variation within the film
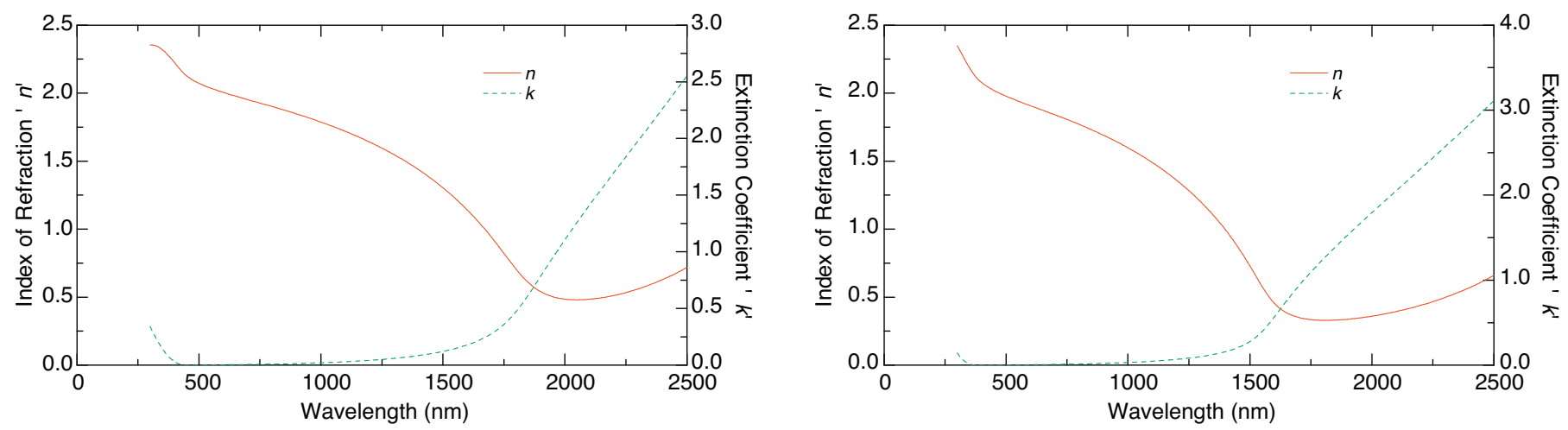

Fig. 2. Dispersion of the optical constants for as-deposited (a) and annealed (b) ITO samples.
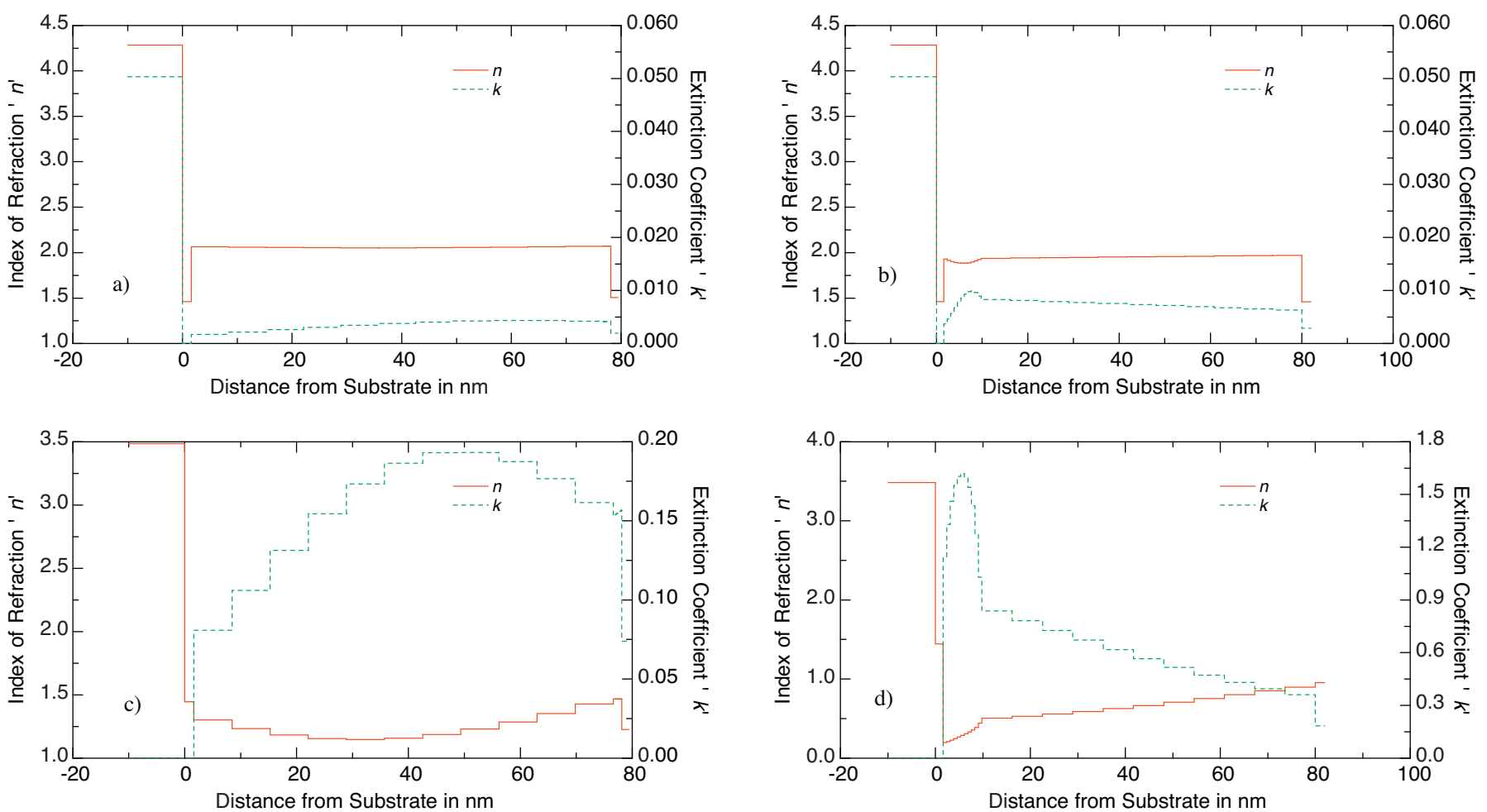

Fig. 3. Optical constants profiles for the thickest among as-deposited (256, left) and annealed samples (241, right): at $500 \mathrm{~nm}$ (a and c) and at $1500 \mathrm{~nm}$ (b and d). 

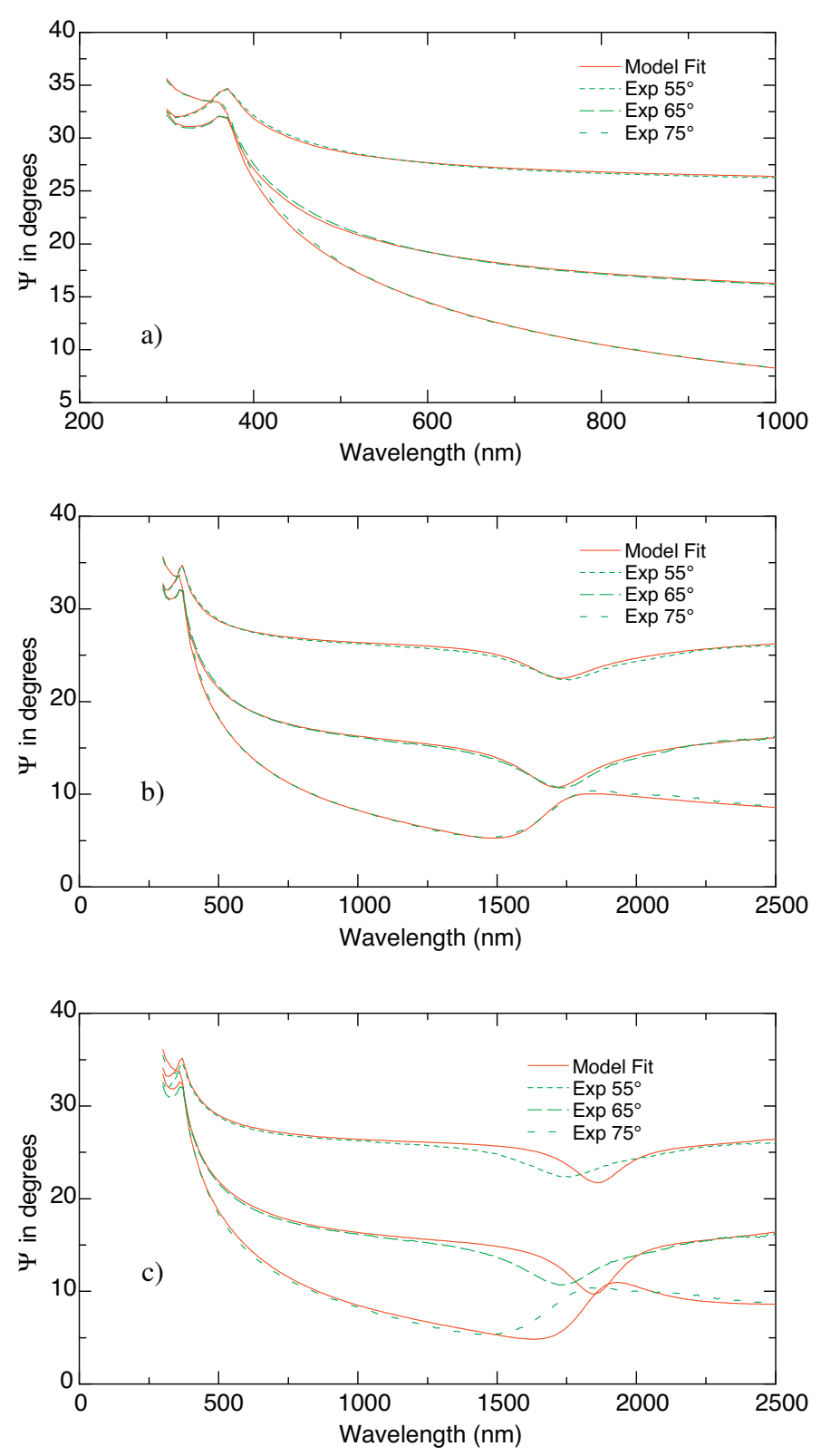

Fig. 4. Fit of the ellipsometric angle $\Psi$ : (a) short range $300-800 \mathrm{~nm}$ within a model of a constantover-thickness optical constants; (b) full range 300-2500 nm within a model of inhomogeneous optical constants and (c) full range $300-2500 \mathrm{~nm}$ within a model of a constantover-thickness optical constants.

depth $z$, when $z$ varies from 0 (substrate) to $d$ (outer surface of the film). Notice that a linear profile of the optical constants is a possible particular case of our generally asymmetric parabolic description $q(z)$.

Before characterization of the ITO layers, the silicon substrate with its native oxide was measured and the oxide thickness was estimated together with dielectric functions of the silicon. The ITO layer roughness was modelled using a surface overlayer with $50 \%$ void inclusions [17]. A sketch of the layered model is shown in Fig. 1.

The two thickest of the layers allow the most reliable in-depth modelling thanks to higher ratio $d / \lambda$, where $d$ is the film thickness. Thus, we used them as a starting point for characterization of the whole sample set and let us consider first these samples as examples of ellipsometric data fit procedure. Fig. 2 shows dispersion of the optical constants of the $\sim 80 \mathrm{~nm}$ thick films. It is clearly seen that the annealing treatment has provoked a blue shift of both the energy gap and the plasma frequency of the material. For further analysis of the sample set it is worth noting right now that there is no physical reason to expect optical dispersion laws different from these two for other samples of each category. For this reason these laws were kept invariable so that only the film and its overlayer thicknesses were fitting parameters together with the geometrical profiles of the optical constants. The geometrical profile of the optical constants of the $\sim 80 \mathrm{~nm}$ thick samples are shown in Fig. 3 at two wavelengths representative for the visible and NIR part of the spectrum. It is rather comprehensible that at $500 \mathrm{~nm}$ ITO behaves like a dielectric so no traces of free carrier distribution within the thickness are expectable. In the vicinity of plasma frequency the situation changes; that is why all the significant for conductivity profiles of $k$ are taken at $1500 \mathrm{~nm}$.

An accurate profiling of the optical constants not only allows the insight of optical and electrical properties of the material under study, but also ensures the correct film thickness determination. It is especially true in the case of ultrathin layers. For the materials with a complex dispersion like ITO has, such a profiling is possible only when the measurement range is sufficiently wide to include the region around the material plasma frequency. This may be illustrated using the example of the sample 250 (about $20 \mathrm{~nm}$ thick as estimated from the deposition rate and run time). Equally good fitting may result for a restricted range $300-800 \mathrm{~nm}$ and for the whole range up to $2500 \mathrm{~nm}$, despite the fact that the first was obtained using invariable-in-the-depth optical constants, Fig. 4a, while the second was possible only when applying a parabolic depth profiling, Fig. $4 \mathrm{~b}$. The correctness of the latter is proved by the values of the film and overlayer thicknesses. In fact, in the first case the resulting film thickness is about $17.3 \mathrm{~nm}$ with an overlayer of $3.6 \mathrm{~nm}$, while in the second case: the film thickness is $18.2 \mathrm{~nm}$, while the overlayer is just $2.6 \mathrm{~nm}$ thick. These last values are better confirmed by the AFM imaging of the sample, see Section 4, as far as the film is a continuous amorphous layer with a low RMS roughness. In addition, another confirmation of the second model's accuracy is the fact that a full-range fit without refractive index variation in depth included in the model results in a high discrepancy both in the UV and especially in the NIR range, Fig. 4c. Corresponding to this last model values of the film and overlayer thicknesses are $15.6 \mathrm{~nm}$ and $6.3 \mathrm{~nm}$, respectively.

It should be noted that in any case the initial approximation for the fit procedure for the ultrathin layers $(d<30 \mathrm{~nm})$ is crucial. It is important to fix the material dispersion type within the model otherwise meaningless values of the layer and overlayer thicknesses will most probably be obtained in a free parameter search.

\section{Results and discussions}

Correct determination of the thickness of the layers thanks to the elaborated approach for ellipsometric characterization of the samples has allowed precise calculation of their resistivity, see last column of Table 1 . The film electrical and morphological properties were compared with the optical constants inhomogeneity within the film thickness.

Fig. 5 shows the extinction coefficient profiles for the samples grown on Si substrates, taken at the wavelength of $1500 \mathrm{~nm}$, which is representative for the conducting properties of the ITO material, i.e. for mobile carriers distribution within the film. It is possible to deduce that the ITO film formation on Si ensures a certain nonuniform distribution of concentration of mobile electrons within the film thickness: for the deposition process under study, the 


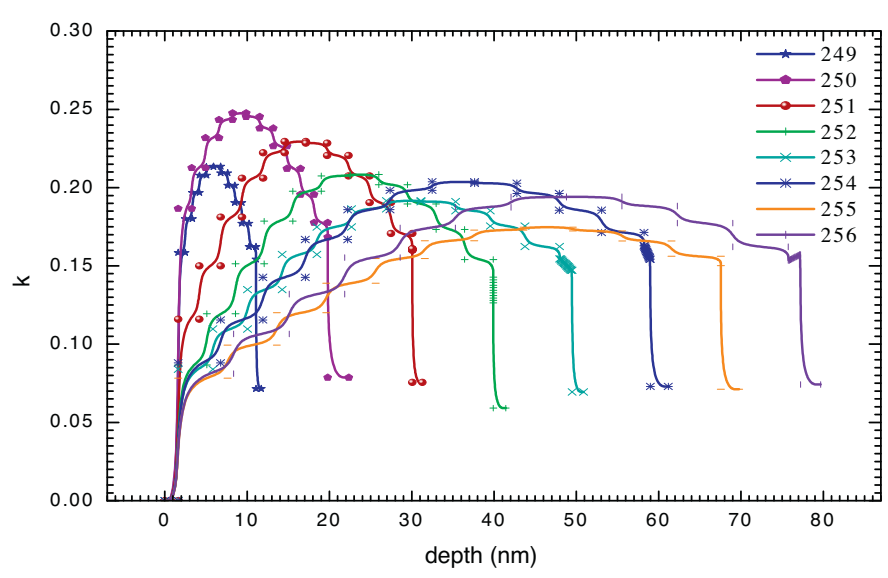

Fig. 5. Thicknesses and extinction coefficient profiles of as-deposited ITO samples.

majority of free carriers and/or those with high mobility in asdeposited ultra-thin ITO films is concentrated at sample half-depth, while their distribution becomes asymmetric for the thicker films, with a maximum located at approximately $40 \mathrm{~nm}$ depth. Moreover, this behaviour is reproducible from sample to sample. Notice that the samples were deposited at low powers for a maximum of $1 \mathrm{~min}$ and 36 s so therefore the substrate heating during deposition was negligible.

For the annealed samples, the material distribution within the film depth changes and becomes far more asymmetric, with extinction coefficient well-pronounced maximum located in the vicinity of the substrate: the main concentration and mobility of the free carriers in ITO film is within the first $10 \mathrm{~nm}$ of the film, see Fig. 6 . Annealing is known to improve ITO performance thanks to diminished disorders and enhanced crystallinity of the material leading to improved electron mobility. A hypothesis on a non-uniform crystal growth and lattice adjustment during annealing may be done. Such an effect occurs for the layers with thicknesses over $40 \mathrm{~nm}$ (with sample 245 showing a "threshold" behaviour), while for thinner films amorphous structure continues to prevail, as confirmed also by AFM measurements. This non-uniformity should follow the temperature distribution inside the film when the substrate is heated, while the outer part of the film results more oxidized compared to its internal fraction.

Fig. 7 shows AFM topography images for the thickest, the thinnest and an intermediate of both as-deposited and annealed

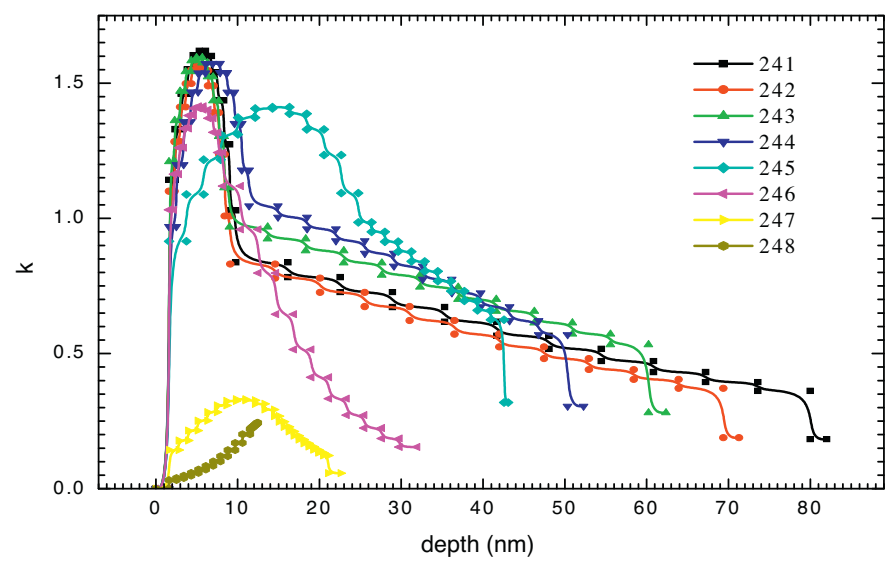

Fig. 6. Thicknesses and extinction coefficient profiles of annealed ITO samples. series of ITO thin films. Obviously, more microstructural features with larger grains formed on the ITO thin films with longer deposition time and thermal annealing. The grains are developed when the thickness of ITO thin film is above $50 \mathrm{~nm}$ (samples 244-241 and 253-256). No observable grains even at largest scale scans should mean that we may consider such films amorphous or at least having prevailing amorphous component (absence of tiny crystals of a few nm-size cannot be proven without structural characterization, of course). This conclusion is not surprising if consider that the samples were deposited without substrate heating. When comparing the morphology images for ITO thin films of samples 249, 254, and 256, larger ITO grains are observed when ITO thickness increases; actually, the ITO lamellae crystals are investigated on the AFM images of the thickest films (samples 241 and 256). This indicates that the larger and well-organized crystal forms when increasing ITO thickness, and crystalline degree increases as well upon film annealing. Another indirect proof of the film structure type is the maintenance of the shape of the extinction coefficient profiles upon annealing for the films thinner than $50 \mathrm{~nm}$ : samples 248-246 have same symmetric $k$-profile like as-deposited samples, and the profiles start loose the symmetry starting from sample 245. This must mean that those films were amorphous when deposited and did not undergo crystallization upon annealing, while sample 245 is presumed to possess a mixture of phases. On the other hand, the films having grains after deposition evolved into more crystalline form confirmed by AFM and by $k$-profile change.

Based on these topography images, surface roughness analysis was done with yielding root mean square (RMS) and average roughness values. Table 2 reports the main roughness parameters of both as-deposited and annealed ITO films on silicon in comparison with ellipsometrically modelled overlayer thickness. It is possible to observe a correlation in these parameters, but the absolute values for surface roughness characterization obtained by AFM and by ellipsometry differ significantly. The main reason for this is that optical probing of the surface in the UV-NIR range reveals the roughness originated by surface features with spatial frequencies which are very different from those AFM refers to. Moreover, modelling of the overlayer as a 50\% Bruggeman-type mixture of air voids and the underlying ITO material is a large-scale approximation over a much bigger surface integration area. Nevertheless, the trend of the overlayer thickness respects the RMS and average roughness values detected by AFM. It is thus concluded that the film roughness can be correctly monitored by ellipsometry, even for ultrathin layers.

Electrical resistivity $\rho_{\text {ott }}$ at optical frequencies may be retrieved from the optical parameters of the film considering that for the optical range when $n \approx k$, at each position $z$ along the film thickness, the conductivity $\sigma(z)$ of the considered sub-layer is related to its extinction coefficient as following [18]:

$\sigma(z)=4 \pi \varepsilon_{0} c \frac{k(z)^{2}}{\lambda}$

where $c$ is the light velocity in vacuum and $\varepsilon_{0}$ is the electric permittivity of vacuum. Therefore, the total film conductivity $\sigma_{\text {ott }}$ may be calculated if consider the parallel connection of all the sub-layers along the film depth:

$\sigma_{\mathrm{ott}}=\frac{1}{d} \int_{0}^{d} \sigma(z) \mathrm{dz}$

so finally,

$\rho_{\mathrm{ott}}=\frac{1}{\sigma_{\mathrm{ott}}}$ 

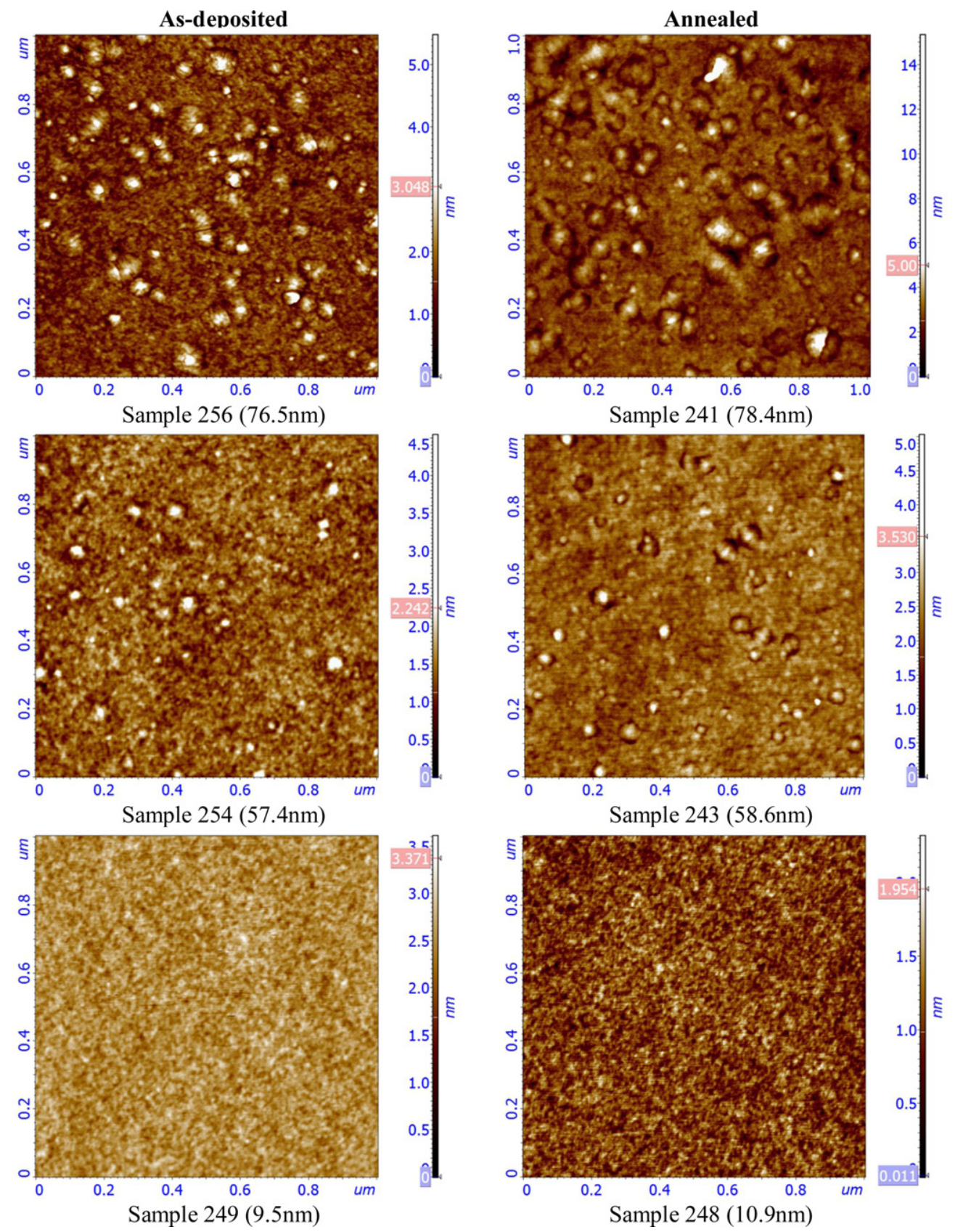

Fig. 7. Topography $1 \mu \mathrm{m} \times 1 \mu \mathrm{m}$ of selected ITO layers on Silicon: as deposited (left) and annealed (right).

The calculated "optical resistivity" for both as-deposited and annealed films is shown in Fig. 8 in comparison with their DC resistivity $\rho_{0}$ measured by the four-probe method. The two types of resistivity have similar behaviour for both sample sets, and in addition the minimum value of resistivity is obtained for the annealed $40 \mathrm{~nm}$ thick sample (sample 245) using these two independent measurement techniques. The minimum at $40-50 \mathrm{~nm}$ is probably connected with the phase mixture nature of these samples (crystalline and amorphous). Sample 247 exhibits no change in the refractive index with annealing and since this implies there is no change in the crystal structures it is not unreasonable for there to be no change in the resistivity, see Table 1 . The samples 248 and 249 evidently have significantly different values of resistivity. However, they are both thin $\sim 10 \mathrm{~nm}$ and as is well known, any slight difference in thickness will result in a disproportionate change in the measured resistivity. Anyway, sample 248 is estimated about $15 \%$ thicker than sample 249 what confirms the tendency in their resistivity values.

Notice that the values of $\rho_{0}$ and $\rho_{\text {ott }}$ must be different because different frequencies are considered. However, if Drude-like conductivity acts in our samples, the trend of the two resistivities should be very similar otherwise it would mean that non-optimal doping depresses the Drude-like conductivity favouring other transport mechanisms caused, for example, by the presence of amorphous regions, localized states etc. These observations corroborate that the optical extinction function is a measure of electrical properties of the ITO films. 
Table 2

RMS and average roughness values from AFM measurements on ITO film versus ellipsometrically determined surface overlayer.

\begin{tabular}{|c|c|c|c|c|c|}
\hline Sample & RMS (5 $\mu \mathrm{m}), \mathrm{nm}$ & $\begin{array}{l}\text { Roughness average } \\
(5 \mu \mathrm{m}), \mathrm{nm}\end{array}$ & RMS (1 $\mu \mathrm{m}), \mathrm{nm}$ & $\begin{array}{l}\text { Roughness average } \\
(1 \mu \mathrm{m}), \mathrm{nm}\end{array}$ & $\begin{array}{l}\text { Ellipsometrically modelled } \\
\text { overlayer, nm }\end{array}$ \\
\hline 241 & 0.638 & 0.403 & 0.639 & 0.417 & 2.02 \\
\hline 242 & 0.416 & 0.24 & 0.428 & 0.303 & 1.9 \\
\hline 243 & 0.379 & 0.259 & 0.293 & 0.219 & 2.2 \\
\hline 244 & 0.389 & 0.245 & 0.239 & 0.185 & 1.9 \\
\hline 245 & 0.47 & 0.241 & 0.215 & 0.168 & 0.5 \\
\hline 246 & 0.236 & 0.143 & 0.228 & 0.18 & 0 \\
\hline 247 & 0.336 & 0.154 & 0.23 & 0.182 & 1.5 \\
\hline 248 & 0.163 & 0.129 & 0.228 & 0.181 & 0 \\
\hline 249 & 0.176 & 0.14 & 0.214 & 0.171 & 0.6 \\
\hline 250 & 0.422 & 0.253 & 0.299 & 0.214 & 2.6 \\
\hline 251 & 0.183 & 0.142 & 0.228 & 0.179 & 1.2 \\
\hline 252 & 0.149 & 0.0942 & 0.133 & 0.103 & 1.5 \\
\hline 253 & 0.189 & 0.146 & 0.26 & 0.203 & 1.4 \\
\hline 254 & 0.244 & 0.178 & 0.248 & 0.185 & 2.2 \\
\hline 255 & 0.328 & 0.207 & 0.383 & 0.281 & 2.2 \\
\hline 256 & 0.528 & 0.254 & 0.372 & 0.265 & 2.4 \\
\hline
\end{tabular}

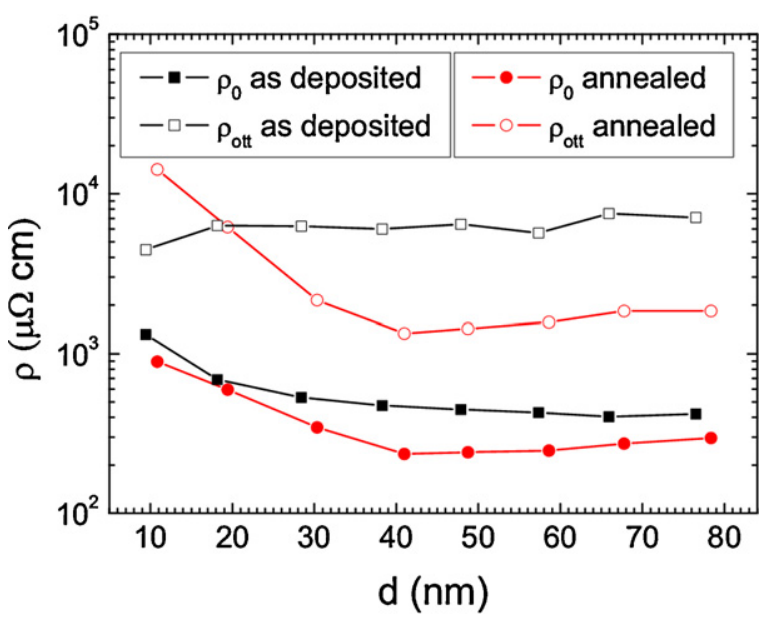

Fig. 8. Thickness dependence of the electric resistivity $\rho_{0}$ and "optical resistivity" $\rho_{\text {ott }}$ at $2000 \mathrm{~nm}$ of the as-deposited and annealed films.

\section{Conclusions}

Film inhomogeneity plays an essential role in ITO characterization allowing correct determination of the film thickness and its optical constants. This is especially true at nanoscale, i.e. for very thin layers (thinner than $\sim 20 \mathrm{~nm}$ ). Moreover, free carriers distribution and mobility along the film depth comprehensible from the shape of the extinction coefficient curve is important for adjustment of the deposition process parameters or those of postdeposition treatment, as well as minimization of the material use once an optimal film thickness with a sufficient number of charge carriers is found.

Film roughness can be correctly monitored by ellipsometry, even for ultrathin layers. Nanoscale effects may be successfully studied by VASE, when the measurement covers the range of essential features of the material and a proper modelling methodology is elaborated.

The ITO film formation on Si ensures a sample to sample reproducible non-uniform distribution of carriers within the film thickness. Detection of re-distribution of the carriers upon annealing was another result of the VASE data modelling.

Finally, this type of analysis has allowed, for the first time to the best of our best knowledge, a systematic study of thickness dependent properties of DC-pulsed sputtered ITO films by a nondestructive optical technique.

\section{Acknowledgement}

This work was performed within the frame of EC project ThinSi, grant agreement no. 241281.

\section{References}

[1] L. Castañeda, Materials Sciences and Applications 2 (2011) 1233-1242.

[2] R.G. Gordon, MRS Bulletin 25 (2000) 52-57.

[3] S. Calan, A.N. Tiwari, Thin Solid Films 518 (2010) 1839-1841.

[4] H.L. Hartnagel, A.L. Das, A.K. Jain, C. Jagadish, Semiconducting Transparent Thin Films, Institute of Physics Publishing, Bristol, 1995.

[5] T. Koida, H. Fujiwara, M. Kondo, Journal of Non-Crystalline Solids 354 (2008) 2805-2808.

[6] K. Ellmer, R. Mientus, Thin Solid Films 516 (2008) 4620-4627.

[7] J. Tate, M.K. Jayaraj, A.D. Dareseke, T. Ulbrich, A.W. Sleight, K.A. Vanaja, R. Nagarajan, J.F. Wager, Thin Solid Films 411 (2002) 119

[8] K.-S. Weissenrieder, J. Mueller, Thin Solid Films 300 (1997) 30-41.

[9] K. Ellmer, R. Mientus, Thin Solid Films 516 (2008) 5829-5835.

[10] D.C. Paine, T. Whitson, D. Janiac, R. Beresford, C.O. Yang, B. Lewis, Journal of Applied Physics 85 (1999) 8445

[11] A. Krasilnikova Sytchkova, M.L. Grilli, S. Boycheva, A. Piegari, Applied Physics A: Materials Science and Processing 89 (2007) 63-72.

[12] D.-H. Kim, M.-R. Park, H.-J. Lee, G.H. Lee, Applied Surface Science 253 (2006) 409-411.

[13] A. Krasilnikova Sytchkova, S. Schutzmann, Bulk and Surface Inhomogeneity of RF Sputtered ITO Films: an Ellipsometric Study in DGaO-PROCEEDINGS, 2009 [ISSN: 1614-8436] http://www.dgao-proceedings.de

[14] R.J. Mendelsberg, G. Garcia, J.D. Milliron, Journal of Applied Physics 111 (2012) 063515.

[15] M. Losurdo, M. Giangregorio, P. Capezzuto, G. Bruno, R. De Rosa, F. Roca, C. Summonte, J. Plá, R. Rizzoli, Journal of Vacuum Science and Technology A 20 (2002) 37-42.

[16] K. Füchsel, U. Schulz, N. Kaiser, A. Tünnermann, Applied Optics 47 (2008) C297-C302.

[17] A. Tikhonravov, M. Trubetskov, A. Krasilnikova, E. Masetti, A. Duparré, E. Quesnel, D. Ristau, Thin Solid Films 397 (2001) 229-237.

[18] (a) F. Bassani, G. Pastore Parravicini, Electronic State and Optical Transition, Pergamon Press, Oxford, 1975;

(b) G. Grosso, G. Pastore Parravicini, Solid State Physics, Academic Press Inc. San Diego, 2000 